\title{
The importance of multimodality in sarcasm detection for sentiment analysis
}

\begin{abstract}
Sentiment analysis is the computational study of opinions given by the users of online media platforms e.g. Twitter, Facebook, Instagram. The output will be in the form of polarity: positive, negative or indifferent. The field has become very useful for the industry as it can feed them the information of what is sought after by their customers in a given time. It has also rapidly became a topic of interest in the research world, for its importance and subjectivity. One of the most challenging issue in sentiment analysis is sarcasm. The existence of sarcasm is mostly ignored by the researchers in the field of sentiment analysis as it is considered to be too complex. Sarcasm is what most researchers regarded as a subset of irony. It is the utterance of positive statement with negative intent. Intent is hard to detect not only for computers but also for humans. The listener is deemed to have a certain degree of background knowledge or context of what the speaker is saying to understand sarcasm. The researches that takes sarcasm into account or solely focuses on sarcasm is in the trend of using context outside the target word for sarcasm detection, and the most popular approach is deep learning. However, both deep learning and context need a lot of features. In this paper, we will look at some researches that focuses on sarcasm detection and their agreement that more than text is needed to properly detect sarcasm. Also in this paper is the trends undergone by sarcasm detection researchers and their proposed techniques.
\end{abstract}

Keyword: Sentiment analysis; Sarcasm detection 